\title{
Traditional Utilization and Harvesting of Medicinal Plants in Mandla District of Madhya Pradesh
}

\author{
Smita Shree Dikshit, Chandra Prakash Kala* \\ Ecosystem \& Environment Management Indian Institute of Forest Management, Nehru Nagar, Bhopal, Madhya Pradesh, INDIA \\ *Corresponding author: cpkala@yahoo.co.uk
}

Received February 26, 2014; Revised March 11, 2014; Accepted March 14, 2014

\begin{abstract}
Utilization and harvesting practices of medicinal plants used by local people of Ghughri block in Mandla district of Madhya Pradesh was studied through questionnaire survey. The study resulted in the documentation of 43 medicinal plants of these $95 \%$ was used by traditional healers for curing various diseases. Tree species were used in maximum cases, followed by herbs and shrubs. Tree bark was the most used plant part for medicinal purpose, followed by root. In all studied villages, the quantities of medicinal plants collection for own consumption was higher than the quantity collected for sale. Species like Embilica officinalis, Terminalia bellirica, Aegle marmelos, Semecarpus anacardium, Buchanania lanzan, Terminalia chebula, and Syzygium cumini were mainly used by local people for own consumption and not for sale. There was almost consistency in collection of medicinal plants across the studied villages however the collection was determined by the season of species availability. Some species being rare and found in limited area was either not collected or collected by a few villagers. The findings of the study are further discussed in the sustainability perspective of medicinal plants and traditional healing systems.
\end{abstract}

Keywords: medicinal plants, harvesting practices, tribal community, Madhya Pradesh, Mandla district, traditional practices

Cite This Article: Smita Shree Dikshit, and Chandra Prakash Kala, “Traditional Utilization and Harvesting of Medicinal Plants in Mandla District of Madhya Pradesh.” Applied Ecology and Environmental Sciences, vol. 2, no. 2 (2014): 48-53. doi: 10.12691/aees-2-2-2.

\section{Introduction}

The medicinal plants play an important role in the socio-cultural, spiritual and health care needs of communities across the world, as they occur in diverse ecosystems [1-7]. The Indian sub-continent is inhabited by large number of tribal communities, and they generally live in the forest and forest fringe areas $[8,9,10,11]$. These communities are mostly unable to access the formal health care systems due to several reasons including the high cost of modern medicine and unavailability of many such facilities in the remote rural areas hence they still fully or partially depend on the medicinal plants of their surrounding areas for health care [12,13].

The Indian state of Madhya Pradesh, wherein the present study was carried out, is inhabited by various tribal communities who are known to have accumulated a great amount of knowledge on the use of various plant species. Various forest types in this Indian state support number of medicinal plants [14,15,16,17]. Mandla district of Madhya Pradesh is one of the tribal dominated districts where the tribal communities mainly depend on forest resources, including medicinal plants for their livelihood and health care [18]. Though attempts have been made in the past by various researchers to document the medicinal plants, as utilized by the forest dwellers, less emphasis was given to study utilization practices and harvesting mechanisms $[16,19]$. Though there are studies available in some parts of Madhya Pradesh [14,16,17,19], no study on the utilization of medicinal plants has been carried out yet in the Ghughri block of the Mandla district of Madhya Pradesh. The present study aims to document the medicinal plants, as utilized by the selected group of local people. Attempt was also made to identify various harvesting and utilization practices of medicinal plants.

\section{Methodology}

\subsection{Study Area}

Madhya Pradesh is located in the central part of India. The variation in the climate, soil and topographic features in the state resulted into diversity of its forests types and floristic composition. The intensive study area - Mandla is a tribal dominated district, situated in the east-central part of Madhya Pradesh. The area of the Mandla district is $8771 \mathrm{~km}^{2}$. According to the 2012 census, population of the district is 779,414 . It has 9 development blocks, 4 tehsil and 1214 villages. It lies between the latitude $22^{\circ} 2^{\prime}$ and $23^{\circ} 22^{\prime}$ north and longitude $80^{\circ} 18^{\prime}$ and $81^{\circ} 50^{\prime}$ east.

Mandla endows with rich forests. Some good forest area of Mandla district falls under Bicchiya tehsil, which constitutes of Bicchiya, Mohgaon, and Mawai and Ghughri development blocks. The present study was 
conducted in the Ghughri block of Mandla district as it has diverse ethnic groups including Gond and Baigas and Medicinal plants wealth, as well. There were 96 villages in Ghughri block out of which three were forest villages and 93 were revenue village. Its total population was 78,690 . Most of the village population was below poverty line [18].

\subsection{Survey Methods}

Literature survey was carried out for compilation of information on medicinal plants of the study area. Secondary information was also gathered from the forest officials of the Ghughri block. Besides, the forest officials at Ghughri were also interviewed on the collection practices of medicinal plants.

\subsubsection{Household Survey}

Out of 96 villages in Ghughri block 4 villages were selected for the collection of first hand information of which 2 were forests (e.g., Sajpani and Gorakhpur), and 2 were revenue villages (e.g., Gajraj and Chattarpur). Forest villages were situated in the forest and were looked after by the forest department while revenue villages were controlled by the revenue department. The extensive survey was conducted in the selected villages for documentation of medicinal plants, the plants part used in different therapies, traditional practices, quantity of medicinal plants collection, and medicinal plants trading. Random sampling was adopted for household survey and minimum 50 households at each selected village were approached for questionnaire survey.

\subsubsection{Selection of the Target groups}

Interviews of knowledgeable persons such as specialized traditional healers were also carried out for documentation of specific knowledge on medicinal plants from each of the selected village. These target groups were selected on the basis of the possession and practice of their indigenous knowledge.

\subsection{Data Analysis}

The primary data as collected on traditional utilization of medicinal plants were analyzed by using parameters such as total medicinal plants collected for own consumption, and for sale. The list of medicinal plants, as used by the local people and traditional healers, was prepared and also a comparative statement was developed on the utilization of medicinal plants between traditional healers and common local people.

\section{Results}

\subsection{Utilization of Medicinal Plants}

A total 43 medicinal plants were documented during the present investigations those were utilized by the villagers and traditional healers of Ghughri block in Mandla district of Madhya Pradesh. Of these except two species 41 species were used by traditional healers and 12 species by common local people residing in the forest as well as non forest villages (Table 1). Maximum tree species were used, followed by herbs and shrubs (Figure 1). Various parts of these plant species were collected. Tree bark was the most used plant part for medicinal purpose (20\%), followed by roots (18\%). Rhizome stem and gum of species were also used for medicinal purpose (Figure 2). Edible fruits of many species such as Aegle marmelos, Annona sqamosa, Cassia fistula, Embilica tsjeriam, Gloriosa superba, Terminalia bellirica and Terminalia chebula were also used for curing diseases. Almost all plant parts of Azadirachta indica, Bauhinia tomentosa, Calotropis gigantea, Euphorbia hirta and Ocimum basilicum were used by traditional healers for curing diseases.



Figure 1. Percentage utilization of various life forms of medicinal plants

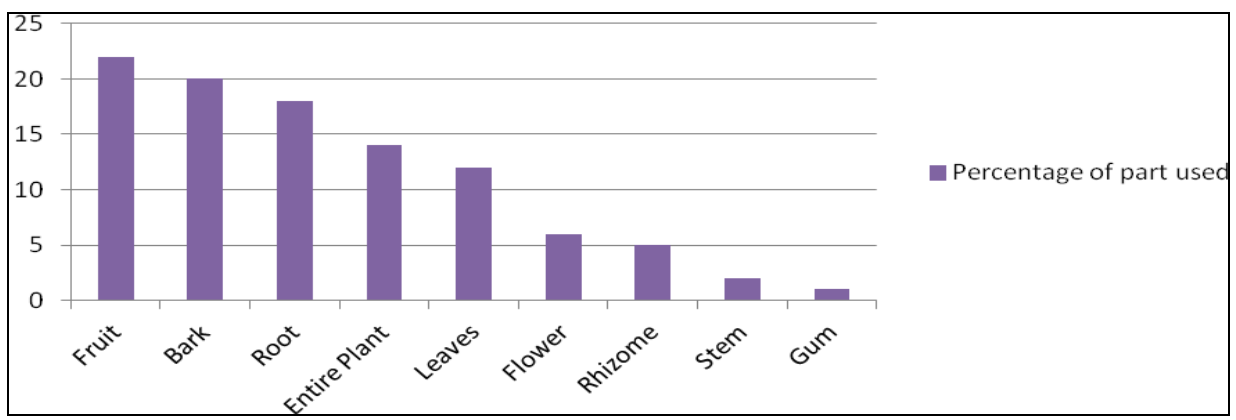

Figure 2. Plant parts of medicinal plants used by the villagers in the study area 
Table 1. Utilization of medicinal plants in the Ghughri block of Mandla district in Madhya Pradesh

\begin{tabular}{|c|c|c|c|c|c|c|c|c|c|}
\hline \multirow[b]{2}{*}{$\begin{array}{l}\text { Sl. } \\
\text { no }\end{array}$} & \multirow[b]{2}{*}{ Plant species } & \multirow[b]{2}{*}{$\begin{array}{l}\text { Local } \\
\text { name }\end{array}$} & \multirow[b]{2}{*}{ Family } & \multicolumn{2}{|c|}{ Availability } & \multicolumn{2}{|c|}{ Utilized by } & \multirow[b]{2}{*}{ Part used } & \multirow[b]{2}{*}{ Indigenous uses } \\
\hline & & & & Locality & Season & $\begin{array}{c}\text { Traditional } \\
\text { healers }\end{array}$ & $\begin{array}{c}\text { Househ } \\
\text { old }\end{array}$ & & \\
\hline 1 & Abrus precatorius & Gumchi & Fabaceae & Forest & Winter & Yes & No & Seed & Stomachache \\
\hline 2 & Acacia catechu & Khair & Fabaceae & Forest & $\begin{array}{c}\text { All } \\
\text { season }\end{array}$ & Yes & No & Bark & Diarrhea, ulceration, antiseptic \\
\hline 3 & Acyranthus aspera & Chirchita & $\begin{array}{c}\text { Amaranthac } \\
\text { eae }\end{array}$ & Forest & $\begin{array}{c}\text { All } \\
\text { season }\end{array}$ & Yes & No & Root & Scorpion and snake sting \\
\hline 4 & Aegal marmelos & Bel & Rutaceae & Forest & $\underset{r}{\text { Summe }}$ & Yes & Yes & Fruit & $\begin{array}{l}\text { Diarrhea, constipation, typhoid, } \\
\text { chronic }\end{array}$ \\
\hline 5 & Annona sqamosa & Chhitafal & Annonaceae & $\begin{array}{c}\text { Cultivati } \\
\text { on }\end{array}$ & $\begin{array}{c}\text { Summe } \\
\mathrm{r}\end{array}$ & Yes & No & Leaves, fruit & Dysentery \\
\hline 6 & Anogeissus latifolia & Dhawa & $\begin{array}{c}\text { Combretace } \\
\text { ae }\end{array}$ & Forest & $\begin{array}{c}\text { All } \\
\text { season }\end{array}$ & No & Yes & Bark, gum & $\begin{array}{l}\text { Gum is having medicinal property } \\
\text { and is edible }\end{array}$ \\
\hline 7 & Argemon maxicana & Katayan & $\begin{array}{c}\text { Papaveracea } \\
\mathrm{e}\end{array}$ & Forest & $\begin{array}{c}\text { All } \\
\text { season }\end{array}$ & Yes & No & Root & $\begin{array}{l}\text { Recovery from heat strokes during } \\
\text { summer }\end{array}$ \\
\hline 8 & $\begin{array}{l}\text { Asparagus } \\
\text { racemosus }\end{array}$ & Satawar & $\begin{array}{c}\text { Asparagace } \\
\text { ae }\end{array}$ & Forest & $\begin{array}{c}\text { All } \\
\text { season }\end{array}$ & Yes & No & Root & Gastro intestinal disorder \\
\hline 9 & Azadirachta indica & Neem & Meliaceae & $\begin{array}{c}\text { Road } \\
\text { side }\end{array}$ & $\begin{array}{c}\text { All } \\
\text { season }\end{array}$ & Yes & No & Entire plant & $\begin{array}{l}\text { Antiseptic periodic, fever, ulcers, } \\
\text { skin disease }\end{array}$ \\
\hline 10 & Bauhinia tomentosa & Kachnar & Fabaceae & Forest & $\begin{array}{c}\text { All } \\
\text { season }\end{array}$ & Yes & No & Entire plant & Dysentery, tonic \\
\hline 11 & $\begin{array}{l}\text { Boerhaavia } \\
\text { chinensis }\end{array}$ & $\begin{array}{c}\text { Patharchi } \\
\text { ta }\end{array}$ & $\begin{array}{c}\text { Nyctangina } \\
\text { ceae }\end{array}$ & Forest & $\begin{array}{c}\text { All } \\
\text { season }\end{array}$ & Yes & No & Entire plant & Jaundice, snake venom, anemia \\
\hline 12 & Buchanania lanzan & Char & $\begin{array}{c}\text { Anacardiace } \\
\text { ae }\end{array}$ & Forest & Spring & Yes & Yes & Fruit & $\begin{array}{c}\text { Used as substitute skin disease, } \\
\text { diarrhea }\end{array}$ \\
\hline 13 & Butea monosperma & Palash & Fabaceae & Forest & $\begin{array}{c}\text { All } \\
\text { season }\end{array}$ & Yes & No & $\begin{array}{c}\text { Seed, bark, } \\
\text { flower }\end{array}$ & Piles, mensuration disorder, diarrhea \\
\hline 14 & Calotropis gigantea & Aak & $\begin{array}{c}\text { Apocynacea } \\
\text { e }\end{array}$ & $\begin{array}{c}\text { Wastela } \\
\text { nd }\end{array}$ & $\begin{array}{c}\text { All } \\
\text { season }\end{array}$ & Yes & No & Entire plant & Fever, cough, skin disease \\
\hline 15 & Cassia fistula & Amaltas & Fabaceae & Forest & $\begin{array}{c}\text { All } \\
\text { season }\end{array}$ & Yes & No & Fruit, flower & Constipation, piles, fever, gastric \\
\hline 16 & Cassia tora & Chakoda & Fabaceae & Forest & Winter & Yes & Yes & Leaves & Cold cough \\
\hline 17 & $\begin{array}{c}\text { Cissus } \\
\text { quadrangularis }\end{array}$ & Hadjod & Vitaceae & Forest & $\begin{array}{c}\text { All } \\
\text { season }\end{array}$ & Yes & No & Leaf, stem & $\begin{array}{l}\text { Prescribed in scurvy for fractures } \\
\text { cardeo-tonic }\end{array}$ \\
\hline 18 & $\begin{array}{c}\text { Curcuma } \\
\text { angustifolia }\end{array}$ & Tikhur & $\begin{array}{c}\text { Zingiberace } \\
\text { ae }\end{array}$ & Forest & $\begin{array}{c}\text { All } \\
\text { season }\end{array}$ & Yes & No & Rhizome & Dysentery, stomachache, wounds \\
\hline 19 & Cylista scariosa & Ban sem & Fabaceae & Forest & $\begin{array}{c}\text { All } \\
\text { season }\end{array}$ & Yes & No & Roots & Dysentery, leucorrhoea \\
\hline 20 & Cyperus scariosus & $\begin{array}{l}\text { Nagarmo } \\
\text { tha }\end{array}$ & Cyperaceae & $\begin{array}{l}\text { River } \\
\text { side }\end{array}$ & $\begin{array}{c}\text { All } \\
\text { season }\end{array}$ & Yes & No & Roots & Stomachache, ulcers \\
\hline 21 & Embelia tsjeriam & $\begin{array}{c}\text { Baibiran } \\
\mathrm{g}\end{array}$ & $\begin{array}{c}\text { Myrsinacea } \\
\mathrm{e}\end{array}$ & Forest & $\begin{array}{c}\text { All } \\
\text { season }\end{array}$ & Yes & No & Fruit & Stomachache, tonic \\
\hline 22 & Emblica officinalis & Amla & $\begin{array}{c}\text { Euphorbiac } \\
\text { eae }\end{array}$ & Forest & Winter & Yes & Yes & Fruit & Edible, laxative \\
\hline 23 & $\begin{array}{l}\text { Eranthemum } \\
\text { purpurascens }\end{array}$ & $\begin{array}{l}\text { Van } \\
\text { Tulsi }\end{array}$ & $\begin{array}{c}\text { Acanthacea } \\
\mathrm{e}\end{array}$ & Forest & Winter & No & Yes & Seed & Cold cough, fever \\
\hline 24 & $\begin{array}{c}\text { Eriolaena } \\
\text { hookeriana }\end{array}$ & Bothi & Malvaceae & Forest & $\begin{array}{c}\text { All } \\
\text { season }\end{array}$ & Yes & No & Root & $\begin{array}{l}\text { Recovery from heat strokes during } \\
\text { summer }\end{array}$ \\
\hline 25 & Euphorbia hirta & Doodhi & $\begin{array}{c}\text { Euphorbiac } \\
\text { eae }\end{array}$ & Forest & $\begin{array}{c}\text { All } \\
\text { season }\end{array}$ & Yes & No & Entire plant & Dysentery, asthma \\
\hline 26 & Gloriosa superba & Kalihari & $\begin{array}{c}\text { Colchicacea } \\
\text { e }\end{array}$ & Forest & Rainy & Yes & No & Rhizome & Cold, ulcers, piles \\
\hline 27 & Grewia tiliaefolia & Dhaman & Malvaceae & Forest & $\begin{array}{c}\text { All } \\
\text { season }\end{array}$ & Yes & No & Bark, stem & Dysentery, cough, pain \\
\hline 28 & Gymnema sylvestre & Gurmar & $\begin{array}{l}\text { Asclepiadac } \\
\text { eae }\end{array}$ & Forest & $\begin{array}{c}\text { All } \\
\text { season }\end{array}$ & Yes & No & Roots, leaves & Stimulant, heart disease, diabetes \\
\hline 29 & Madhuca indica & Mahua & Sapotaceae & Forest & $\begin{array}{c}\text { Summe } \\
\mathrm{r}\end{array}$ & Yes & Yes & Fruit, flower & Bleeding gums, ulcers, diabetes \\
\hline 30 & Mucuna pruriens & Kiwanch & Fabaceae & Forest & $\begin{array}{c}\text { All } \\
\text { season }\end{array}$ & Yes & No & Seed, roots & Tonic, stimulant, diuretic, diarrhea \\
\hline 31 & Ocimum basilicum & Van tulsa & Lamiacea & $\begin{array}{c}\text { Wastela } \\
\text { nd }\end{array}$ & $\begin{array}{c}\text { All } \\
\text { season }\end{array}$ & Yes & No & Entire plant & Fever, cough, constipation \\
\hline 32 & Ricinus communis & $\begin{array}{l}\text { Andi,Ara } \\
\text { nd }\end{array}$ & $\begin{array}{c}\text { Euphorbiac } \\
\text { eae }\end{array}$ & Forest & $\begin{array}{c}\text { All } \\
\text { season }\end{array}$ & Yes & No & Roots, seeds & Scorpion and snake sting \\
\hline 33 & Schleichera oleosa & Kusum & Sapindaceae & Forest & $\begin{array}{c}\text { All } \\
\text { season }\end{array}$ & Yes & Yes & Seed oil, bark & $\begin{array}{l}\text { Skin diseases, rheumatic pain, } \\
\text { itches, ulcers }\end{array}$ \\
\hline 34 & $\begin{array}{l}\text { Semecarpus } \\
\text { anacardium }\end{array}$ & Bhelwa & $\begin{array}{c}\text { Anacardiace } \\
\text { ae }\end{array}$ & Forest & Spring & Yes & Yes & Bark, seed & $\begin{array}{c}\text { Asthma, diarrhea, dysentery, } \\
\text { rheumatism }\end{array}$ \\
\hline 35 & Soymida febrifuga & Rohan & Meliaceae & Forest & $\begin{array}{c}\text { All } \\
\text { season }\end{array}$ & Yes & No & Bark, resin & $\begin{array}{c}\text { Diarrhea, dysentery, fever, tonic, } \\
\text { malaria }\end{array}$ \\
\hline 36 & Sterculia urens & Kullu & Malvaceae & Forest & $\begin{array}{c}\text { All } \\
\text { season }\end{array}$ & Yes & No & Bark, gum & $\begin{array}{c}\text { Facilitate delivery, laxative, dental } \\
\text { fixture }\end{array}$ \\
\hline 37 & Syzygium cumini & Jamun & Myrtaceae & Forest & $\begin{array}{c}\text { Summe } \\
\mathrm{r}\end{array}$ & Yes & Yes & Fruit & Constipation, skin diseases, diarrhea \\
\hline 38 & Tephrosia purpurea & $\begin{array}{c}\text { Sarphonk } \\
\text { a } \\
\end{array}$ & Fabaceae & Forest & $\begin{array}{c}\text { All } \\
\text { season }\end{array}$ & Yes & No & Leaves & Tonic, laxative, diarrhea \\
\hline 39 & Terminalia arjuna & Arjuna & $\begin{array}{c}\text { Combretace } \\
\text { ae }\end{array}$ & $\begin{array}{l}\text { River } \\
\text { side }\end{array}$ & $\begin{array}{c}\text { All } \\
\text { season }\end{array}$ & Yes & No & Bark & Astringent, blood pressure \\
\hline 40 & Terminalia bellirica & Baheda & $\begin{array}{c}\text { Combretace } \\
\text { ae }\end{array}$ & Forest & Spring & Yes & Yes & Fruit & Tonic, laxative \\
\hline 41 & Terminalia chebula & Harra & $\begin{array}{c}\text { Combretace } \\
\text { ae }\end{array}$ & Forest & Spring & Yes & Yes & Fruit & Tonic, laxative, diuretic \\
\hline 42 & $\begin{array}{l}\text { Woodfordia } \\
\text { fruticosa }\end{array}$ & Surteli & Lythraceae & Forest & $\begin{array}{c}\text { All } \\
\text { season }\end{array}$ & Yes & No & Entire plant & Diarrhea, dysentery \\
\hline 43 & Zizyphus mauritiana & Ber & $\begin{array}{c}\text { Rhamnacea } \\
\text { e }\end{array}$ & Forest & Winter & Yes & No & $\begin{array}{c}\text { Bark, fruit, } \\
\text { seed }\end{array}$ & Diarrhea, dysentery \\
\hline
\end{tabular}


Table 2. Medicinal plants utilized by households of study area for own consumption and sale

\begin{tabular}{|c|c|c|c|c|c|c|c|c|c|}
\hline S & Species collected & \multicolumn{8}{|c|}{$\%$ of collection by household } \\
\hline & & Sajpani & & Gorakhpur & & Gajraj & & Chattarpur & \\
\hline & & $\begin{array}{c}\text { For own } \\
\text { consumption }\end{array}$ & $\begin{array}{l}\text { For } \\
\text { sale }\end{array}$ & $\begin{array}{c}\text { For own } \\
\text { consumption }\end{array}$ & $\begin{array}{l}\text { For } \\
\text { sale }\end{array}$ & $\begin{array}{c}\text { For own } \\
\text { consumption }\end{array}$ & $\begin{array}{l}\text { For } \\
\text { sale }\end{array}$ & $\begin{array}{c}\text { For own } \\
\text { consumption }\end{array}$ & $\begin{array}{l}\text { For } \\
\text { sale }\end{array}$ \\
\hline 1 & Aegle marmelos & $80 \%$ & 0 & $100 \%$ & 0 & $90 \%$ & 0 & $95 \%$ & 0 \\
\hline 2 & Anogeissus latifolia & $100 \%$ & 0 & $100 \%$ & 0 & $100 \%$ & 0 & $100 \%$ & 0 \\
\hline 3 & Buchanania lanzan & $30 \%$ & 0 & $70 \%$ & $8 \%$ & $99 \%$ & 0 & $88 \%$ & 0 \\
\hline 4 & Cassia tora & $100 \%$ & $100 \%$ & $100 \%$ & $100 \%$ & $90 \%$ & $80 \%$ & $90 \%$ & $75 \%$ \\
\hline 5 & Embilica officinalis & $40 \%$ & 0 & $50 \%$ & 0 & $80 \%$ & 0 & $70 \%$ & 0 \\
\hline 6 & $\begin{array}{l}\text { Eranthemum } \\
\text { purpurascens }\end{array}$ & $0 \%$ & $100 \%$ & $0 \%$ & $80 \%$ & 0 & $4 \%$ & 0 & 0 \\
\hline 7 & Madhuca indica & $100 \%$ & $100 \%$ & $100 \%$ & $100 \%$ & $100 \%$ & $100 \%$ & $100 \%$ & $100 \%$ \\
\hline 8 & Schleichera oleosa & $16 \%$ & 0 & $0 \%$ & 0 & 0 & 0 & 0 & 0 \\
\hline 9 & Semecarpus anacardium & $100 \%$ & $20 \%$ & $100 \%$ & $12 \%$ & $100 \%$ & 0 & $98 \%$ & 0 \\
\hline $\begin{array}{l}1 \\
0\end{array}$ & Syzgium cumini & $60 \%$ & 0 & $70 \%$ & 0 & $80 \%$ & 0 & $84 \%$ & 0 \\
\hline $\begin{array}{l}1 \\
1\end{array}$ & Terminalia bellirica & $100 \%$ & 0 & $100 \%$ & 0 & $70 \%$ & 0 & $92 \%$ & 0 \\
\hline $\begin{array}{l}1 \\
2\end{array}$ & Terminalia chebula & $100 \%$ & 0 & $100 \%$ & 0 & $70 \%$ & 0 & $88 \%$ & 0 \\
\hline
\end{tabular}

\subsection{Harvesting and Post Harvesting of} Medicinal Plants

Some medicinal plants were collected by local people for their own consumption while some medicinal plants were collected for both sale and own consumption (Table 2). In all studied villages, the quantities of medicinal plants collection for own consumption is higher than the quantity collected for sale. Terminalia tomentosa, Anogeissus latifolia and Ougenia ooginensis though having medicinal properties yet the maximum quantity of these species were utilized as fuel wood. Species like Embilica officinalis, Terminalia bellirica, Aegle marmelos, Semecarpus anacardium, Buchanania lanzan, Terminalia chebula, and Syzygium cumini were mainly used for own consumption and not for sale.

The local people generally preferred to go for selling the collected medicinal plants by weekly basis due to poor road connectivity, especially in the forest villages which were situated deep inside the forests. Species like Madhuca indica, Cassia tora and Eranthemum purpurascens were collected and sold out either through forest department or sometimes directly in the market. In
Chattarpur, Eranthemum purpurascens is not being collected for sale. Thus, here in this village out of the 4 species which is used as a source of income only 3 species were sold out on a weekly basis. Out of the studied villages, Gorakhpur was the only one which collected Buchanania lanzan for own consumption as well as for selling.

There was almost uniform pattern in collection of medicinal plants across the studied villages however the collection was determined by the season of specific species availability (Table 3 ). Some species being rare and found in limited area was either not collected or collected by a few villages. Buchanania lanzan was collected and utilized by the villagers of Gorakhpur for their own consumption as well as for selling whereas the villagers of Sajpani did not report its collection for sale. Since tree species was collected in majority of cases (52\%) and trees being available round the year, the collection from such species remained operational in all seasons (Figure 3). The collection was done from $68 \%$ of species in all seasons, followed by summer (11\%), winter (10\%), spring (9\%) and rainy seasons (2\%). Being annual the occurrence of some species was determined by the season such as Cassia tora.

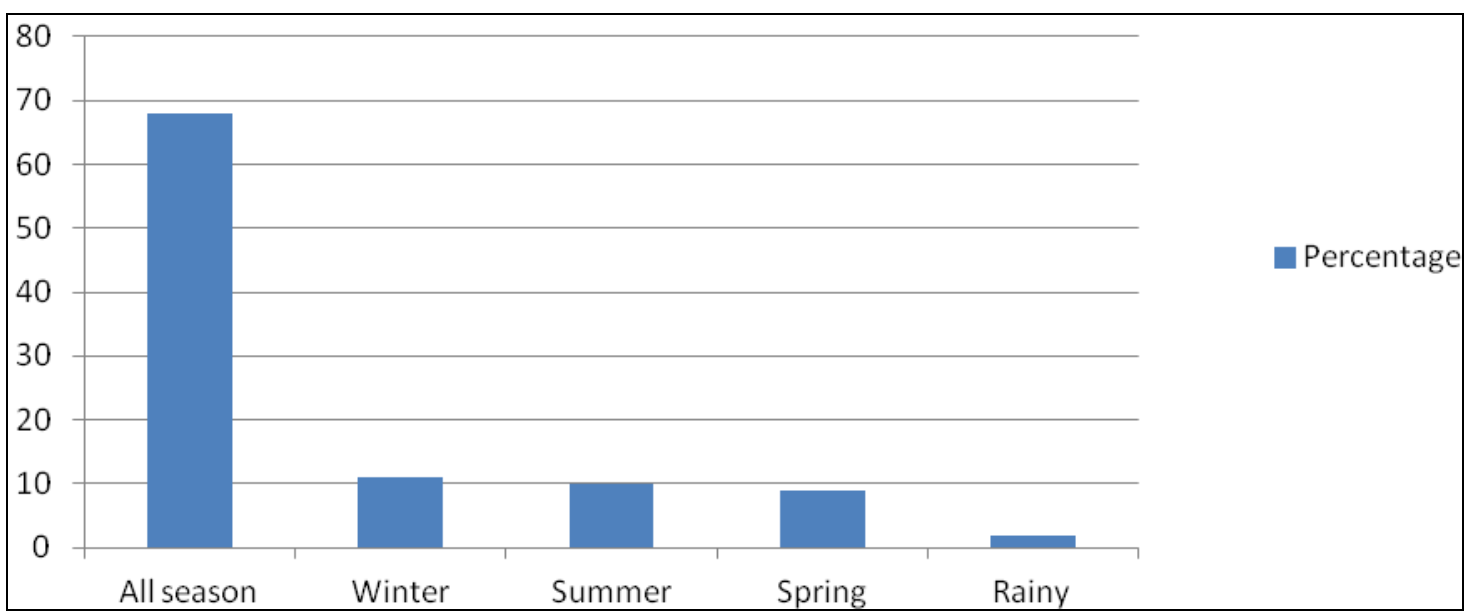

Figure 3. Seasonal availability (in percentage) of medicinal plants utilized by the villagers 
Table 3. Seasonal harvesting pattern of medicinal plants utilized by the households in the studied villages of Mandla district

\begin{tabular}{|c|c|c|c|c|c|c|c|c|c|c|c|c|c|}
\hline \multirow[t]{2}{*}{ Sn } & \multirow[t]{2}{*}{ Local name } & \multicolumn{12}{|c|}{ Season of harvesting } \\
\hline & & Jan & Feb & Mar & Apr & May & Jun & Jul & Aug & Sep & Oct & Nov & Dec \\
\hline 1 & Amla & & & & & & & & & & & & \\
\hline 2 & Baheda & & & & & & & & & & & & \\
\hline 3 & Bel & & & & & & & & & & & & \\
\hline 4 & Bhelwa & & & & & & & & & & & & \\
\hline 5 & Chakoda & & & & & & & & & & & & \\
\hline 6 & Char & & & & & & & & & & & & \\
\hline 7 & Dhawa & & & & & & & & & & & & \\
\hline 8 & Harra & & & & & & & & & & & & \\
\hline 9 & Jamun & & & & & & & & & & & & \\
\hline 10 & Kusum & & & & & & & & & & & & \\
\hline 11 & Mahua & & & & & & & & & & & & \\
\hline 12 & Van Tulsi & & & & & & & & & & & & \\
\hline
\end{tabular}

\section{Discussion}

The local people of Ghughri block in Mandla district have been using medicinal plants growing in their surrounding village forests for curing various human diseases from antiquity. Though some of the medicinal plants were being collected and used by almost every household in the study area, there were specialized knowledge holders and traditional healers who used 95\% of medicinal plants documented during the survey. The specialized knowledge holders mainly dealt to cure some specific disease not more than one or two such as snake bite, scorpion bite, malaria and jaundice whereas the commonly known diseases were cured by rest of the traditional healers. Of the total species used for curing diseases, 8 species such as Aegle marmelos, Buchanania lanzan, Cassia tora, Terminalia bellirica, Terminalia chebula, Syzygium cumini, Semecarpus anacardium and Schleichera oleosa were used by both household member and traditional healers, as well.

On an average 12 people per month visited traditional healers for treatments. The traditional healers and specialized knowledge holders, as well, were mainly visited for curing snake bite, scorpion bite, jaundice, delivery, skin diseases, diabetes, heart diseases, fever, malaria, diarrhea and dysentery. Some species were used for curing specific disease such as Annona sqamosa was used for curing dysentery, Acyranthus aspera was used in scorpion and snake bite, Abrus precatorious was used for stomachache and Argemon maxicana was used for getting recovered from heat stroke. For the treatment of diarrhea, dysentery and constipation many species were discovered by healers including Aegle marmelos, Acacia catechu, Mucuna pruriens, Soymida febrifuga, Syzygium cumini, Woodfordia fruticosa and Zizyphus mauritiana. The healers also mixed up sugar and honey in preparing some herbal formulation.

Medicinal plants were collected mostly from the wild. Only a few households reported cultivation of some medicinal plant species. The collection pattern was almost similar across the villages. The collection practices of medicinal plants were also influenced by market forces hence premature collection was also rampant. The fruits of Embilica officinalis mature after December [4,19], but due to high demand in industries and intense competition among collectors, the harvesting was started in October only (Table 3). Lopping of branches of some medicinally important trees were also observed in the study area.
The local people had less concern about proper harvesting period of medicinal plants. The seasonal harvesting of medicinal plants in studied villages indicates that Embilica officinalis, Buchanania lanzan, Terminalia chebula and Terminalia bellirica were subjected to premature harvesting for collection of fruits. The actual harvesting season of these species is April to May, but people collected them in March and sometime even earlier. This suggests that villagers were not following the proper and traditional harvesting practices. The results of present study on harvesting practices do not fully corroborate with a study of Chauhan [20], which concluded that tribal people were aware of the necessity of the preservation and propagation of species. The study reveals the pressure on medicinal plants, due to illegal lopping and felling of important plant species. Overexploitation of forest by cutting and uprooting trees for easy and quick collection of medicinal plants may lead negative impacts on medicinal plants as well as the traditional health care system.

\section{Acknowledgement}

The authors thank villagers of Sajpani, Gorakhpur, Gajraj and Chattarpur of Mandla district for their cooperation and help during the fieldwork. The state forest department of Madhya Pradesh is acknowledged for extending support during the survey.

\section{References}

[1] Raven, P.H. 1998. Medicinal plants and global sustainability: The canary in the coal mine. In Medicinal Plants: A Global Heritage. Proceedings of the International conference on medicinal plants for survival. International Development Research Center, New Delhi, pp. 14-18.

[2] Chopra, K. 1993. The value of non-timber forest products: an estimate from India. Economic Botany, 47: 251-257.

[3] Kala, C.P. 2003. Commercial exploitation and conservation status of high value medicinal plants across the borderline of India and Nepal in Pithoragarh. Indian Forester, 129 (1): 80-84.

[4] Kala, C.P. 2009. Aboriginal uses and management of ethnobotanical species in deciduous forests of Chhattisgarh state in India. Journal of Ethnobiology and Ethnomedicine, 5: 1-12. http://www.ethnobiomed.com/content/5/1/20

[5] Bhojvaid, P. 2003. Medicinal Plants Based Forest Management. Indian Forester, 129 (1): 25-36.

[6] Hamilton, A.C. 2004. Medicinal plants, conservation and livelihoods. Biodiversity and Conservation 13, 1477-1517. 
[7] Hoareau, L. 1999. Medicinal Plants: A Re-emerging Health Aid. Electronic Journal of Biotechnology, 2 (2): 56-70.

[8] Jagtap, S.D., Deokule, S.S. and Bhosle, S.V. 2006. Some unique ethnomedicinal uses of plants used by the Korku tribe of Amravati district of Maharashtra, India. Journal of ethnopharmacology, 107 (3): 463-469.

[9] Kala, C.P. and Ratajc, P. 2012. High altitude biodiversity of the Alps and the Himalayas: ethnobotany, plant distribution and conservation perspective. Biodiversity and Conservation. Vol. 21, Number 4, pages 1115-1126.

[10] Kala, C. P. 2005. Ethanomedicinal Botany of the Apatani in the Eastern Himalayan region of India. Journal of Ethanobiology and Ethno medicine, 1 (11): 1-15.

[11] Nag A., Galav, P. and Katewa, S.S. 2007. Indigenous animal healthcare practices from Udaipur district, Rajasthan. Indian Journal of Traditional Knowledge, 6 (4): 583-588.

[12] Kala, C.P. 2005. Current status of medicinal plants used by traditional Vaidyas in Uttaranchal state of India. Ethnobotany Research and Applications, 3: 267-278.

[13] Kala, C.P. 2011. Indigenous Uses and sustainable Harvesting of Trees by Local People in Panchmarhi Biosphere reserve of India. International Journal of Medicinal and Aromatic Plants, 1 (2): $153-161$.
[14] Mahajan, S.K. 2007. Traditional herbal remedies among the tribes of Bijagarh of West Nimar district, Madhya Pradesh. Indian Journal of Traditional Knowledge, 6 (2): 375-377.

[15] Kala, C.P. 2010. Home gardens and management of key species in the Pachmarhi Biosphere Reserve of India. Journal of Biodiversity, 1 (2): 111-117.

[16] Kala, C.P. 2012. Traditional ecological knowledge and conservation of ethnobotanical species in the buffer zone of Pachmarhi Biosphere Reserve, Madhya Pradesh. Indian Institute of Forest Management, Bhopal, Madhya Pradesh. 194 pp.

[17] Yadav, M. and Misra, S. 2012. Sustainable development: a role for market information systems for non - timber forest products. Sustainable Development, 20 (2): 128-140.

[18] Shrivastava, R.K. 2010. Working Plan of East Mandla Forest Division for 2010-11 to 2019-2020. Jabalpur, Madhya Pradesh: Madhya Pradesh Government, Forest Department.

[19] Prasad, R., Kotwal, P. and Mishra, M. 2002. Impact of Harvesting of Embelica officinalis (Aonla) on Natural Regenaration, Health, Vitality and Ecosystem in Central Indian Forest. Journal of Sustainable Forestry, 14 (4): 1-12.

[20] Chauhan, N.S. 1999. Medicinal and Aromatic Plants of Himachal Pradesh. New Delhi: Indus Publishing Company. 